\title{
An improved evidence theory-based trust model for multiagent resource allocation
}

\author{
Ningkui Wang*, Hayfa Zgaya-biau, Philippe Mathieu, Slim Hammadi \\ Univ. Lille, CNRS, Centrale Lille, UMR 9189-CRIStAL \\ Centre de Recherche en Informatique Signal et Automatique de Lille, F-59000 Lille, France. \\ Email: ningkui.wang@centralelille.fr
}

\begin{abstract}
In a resource allocation system, resource suppliers and customers can be naturally modelled as autonomous and interactive entities. In this context, we propose in this paper a multiagent system architecture based on trust and honesty concepts between agents in order to synchronize resource allocation in a distributed environment. Indeed, agents use several means in order to allocate resources efficiently as dialogue, adaptability, cooperation, collaboration and even negotiation in addition to the notion of trust. So, individual agents have to evaluate the trustworthiness of others to select those to interact with. Moreover, resource inadequacies can exist in the resource allocation systems, and it is difficult to meet the resource requirements of all agents simultaneously. To overcome these difficulties, we propose a distributed multiagent resource allocation system that emphasizes the issues of agents trust and resource inadequacies, which is called MARA-T\&R. In this system, the evidence theory is improved thanks to the Deng entropy to estimate the trustworthiness of agents. Additionally, we use the concept of reservations to solve the problem of resource inadequacies. Our simulation results highlight the excellent performances of this improved trust model and the efficiency of the proposed MARA-T\&R system for resource allocation.
\end{abstract}

Index Terms-Multiagent system, Resource allocation, Trust, Uncertainty, Resource inadequacy

\section{INTRODUCTION}

Over the past few decades, resource allocation has been a hot issue that received much attention [1]. With the development of multiagent systems (MASs), these technics have taken a new dimension. Distributing a number of resources amongst various agents is known as MARA (multiagent resource allocation) [1]. MARA has been used in a wide range of applications, including, but not limited to public transportation, logistics, exploitation of earth observation satellites, or industrial procurement.

In a distributed environment, agents communicate with each other using particular rules and protocols to allocate resources. In this context, numerous models for agent communication have emerged to distribute resources, including negotiation, coordination, and collaboration. However, several challenges arise together linked to these protocols. For example, an agent in a distributed system makes decisions by investigating the received local information for its intended purpose, which means that the priority is to maximize its private utility. For this reason, it is difficult to get rid of some misleading resource information delivered by unfriendly agents. At the same time, because of the limited capability of storage and calculation, agents have to trust the information they receive to minimize hazards and to achieve more personal goals.

Trust, a concept of human beings, is often used to capture the reliability or honesty of agents in MASs [2], which makes sense to improve the quality of interactions. In general, there are four main models associated with trust systems: Logical models describe how one agent trust another according to mathematical logic; Social-cognitive models estimate the trustworthiness by taking inspiration from human psychology; Organizational models capture trust by personal relationships in the system considered, and numerical models understand trust from the perspective of mathematical probability [3], [4]. We focus on the numerical and social-cognitive models in this paper. To obtain the trustworthiness of an agent, the numerical models need to collect some information concerning the potential interaction. Typically, they are direct trust, which is based on the previous experience from its direct interactions [5]; Indirect reputation, which is based on the recommendation provided by third-party agents [4], [6].

Numerous methodologies have been employed to process the collected information in the context of numerical models. Evidence theory, also referred to as the theory of belief functions or Dempster-Shafer theory (DST), has also been adopted [7], [8]. In the system of multiagent, an agent hires the basic probability of assignment (BPA) to represent a source of information provided by other agents. However, two issues need to be thoroughly stressed; that is, the reliability of previous experience might fade over time, as well as processing conflicts between BPAs. Our goal is therefore to improve these aspects for trust estimation in MASs.

Accounting for multiple agents pursuing first-rate resources, we also adopt the concept of reservations to emphasize the expected future changes and fairness in MARA systems [9], [10]. In summary, this paper makes two main contributions, namely, to propose an improved DST trust models in MAS, and resolve resource allocation in a multiagent context thanks to the improved trust model and the concept of reservation. The remainder of the paper is organized as follows. In section 2 , we highlight state of the art about some related works, and some preliminaries are discussed in section 3 . The proposed model is presented in section 4. Simulations and results are then discussed in section 5. Conclusions and perspectives are included in the last section. 


\section{RELATED WORKS}

The literature is rich with different approaches to trust detection in MAS, DST has also been employed [11], [12]. In [13], DST is used for distributed reputation management of electronic commerce. The method relies on either direct trust or indirect reputation, and indirect reputation is not necessary if the direct trust is obtained. Meanwhile, Dempster's combination rule is directly applied to integrate multiple materials. Swift trust in a virtual temporary system based on DST is recognized in the literature [7]. The proposed method classifies the swift trust in three sub-objectives (the vulnerability, uncertainty, and venture) supported by five influencing factors, and Dempster's rule of combination is employed to fuse all influencing factors. In the literature [14], trust transitivity is stressed by the DST. They described the trust relationship considering uncertainty and established a trust transition model based on the trust features and trust relationship types. In [8], the authors utilized DST to address the network security problem in wireless sensor networks. The BPA is defined by some trust factors, such as the received packets rate, packets forwarding rate. The evidence similarity is processed as weights to modify evidence, and the modified parts are designated as unknown. The proposed method can be used to identify malicious nodes. However, uncertainty, as well as the property that the reliability of evidence vanishing over time, are not emphasized.

The aforementioned DST-based trust models have both advantages and disadvantages. In terms of limitations, the property that evidence reliability vanishing over time is not well stressed when generating BPAs. In addition, the standard Dempster's combination rule is not applicable directly when combining conflicting evidence. Recently, evidence distance and entropy-based models have been used to combine conflicting evidence outside of the MAS community [15]-[18]. However, we realize that the assigned weight is directly proportional to the entropy of the evidence (the higher the entropy, the greater the weight) in data fusion models [16], [17], [19]. We argue that great weights are assigned to the high-entropy evidence is inappropriate. Additionally, to the best of our knowledge, to cope with the combination of conflict evidence with the amount of information conveyed by BPAs has rarely been stressed, especially in the field of trust estimation. We therefore propose an improved DST-based trust estimation model (ITEM) in MASs, mainly to emphasize the two limitations as discussed. Firstly, we propose to generate direct trust considering interaction orders, with which the fading property is stated. Also, we improve the entropy-based weighted approach when combing evidence.

\section{DEMPSTER-ShAFER THEORY OF EVIDENCE}

DST, proposed by Dempster and Shafer to handle uncertainty [11], [12], has a wide range applications in risk assessments, medical diagnosis, and target recognition. It is a typical method of uncertain information fusion [20]. Some preliminaries are introduced below.

definition 1: The frame of discernment and BPAs
DST is defined in the frame of discernment denoted by $\Omega$ which consists of $n$ mutually exclusive and collectively exhaustive elements. $2^{\Omega}$ is the set of all subsets of $\Omega$. Mathematically, a basic probability assignment (BPA, also known as mass function) is $2^{\Omega} \rightarrow[0,1]$, with $\sum_{A \in 2^{\Omega}} m(A)=1$ and $m(\emptyset)=0$ satisfied, where $\emptyset$ is the empty set, the non-zero element $A$ is named focal element, the set of all focal elements is called core. Given two BPAs $m_{1}$ and $m_{2}$, Dempster's combination rule $m=m_{1} \oplus m_{2}$ is used to combine them.

definition 2: Dempster's combination rule

$$
m(A)= \begin{cases}\frac{1}{1-K} \sum_{B \cap C=A} m_{1}(B) m_{2}(C), & A \neq \emptyset ; \\ 0, & A=\emptyset ;\end{cases}
$$

with $K=\sum_{B \cap C=\emptyset} m_{1}(B) m_{2}(C), A, B$ and $C$ are elements in $2^{\Omega}$. The normalization constant $K$ shows the conflicting degree between defined BPAs.

It has to be stressed that Dempster's combination rule makes sense only when $K<1$, otherwise it is meaningless. To put it another way, when BPAs are in high conflict, irrational results might be generated [21]. In the previous studies, there are two main methods to deal with highly conflicting evidence combination, namely modifying the combination rule [22] and preprocessing data [23], [24]. Han et al. modified weighted average by evidence similarities is often used for data preprocessing [24]. In this paper, we use the amount of information contained in BPAs to manage high-conflict evidence combination. Entropy of DST is the methodology proposed to measure the amount of information conveyed by BPAs [25], [26]. In what follows, some basic conceptions related to entropy are introduced.

\section{definition 3: Entropy of DST}

Entropy is a measure of uncertainty and disorder that comes from physics [27]. Shannon entropy is often implemented to estimate the average amount of information carried by a message in the context of probability theory [28]. As a generation of probability theory, many definitions of entropy of DST have arisen, for instance, the definitions proposed by Smets [29], Yager [30], Deng [25] and so on [17], [31]. We use Deng entropy to capture the uncertainty of BPAs. Given a BPA $m_{i}$, Deng entropy [25], [26] is defined

$$
E_{d}\left(m_{i}\right)=-\sum_{F \subseteq 2^{\Omega}} m_{i}(F) \log _{2}\left(\frac{m_{i}(F)}{2^{|F|}-1}\right) .
$$

where $F$ is the focal element in the BPA and $|F|$ is the cardinality of $F$.

\section{MARA-T\&R SYSTEM}

In this part, we present the MARA-T\&R system, which consists of two principal parts. One is to quantify the reliability of agents based on the improved DST, and another is to allocate resources based on agent trustworthiness and the concept of reservation. In what follows, we first briefly introduce the system of multiagents. 


\section{A. Multiagent system}

The role of an agent can be interpreted as a computer program that acts as a user or other program. Agents may generate tasks, dialogue, perform actions, learn, and adapt to the changing environments. A MAS is a computerized system of multiple agents and their environments. When discussing resource allocation in a MAS, both resource suppliers and customers are modelled as intelligent agents, they dialogue, cooperate and collaborate on resource allocation [32].

Our proposed MARA-T\&R system consists of multiple agents, each of which has general features such as unique identity (ID). Of course, agents also have other specific-purpose capacities such as communication, memory and reasoning. We particularly stress the allocation of touchable and visible resources in a distributed MAS. An agent can be either a resource supplier or a resource customer, and sometimes, it could be both a resource supplier and a resource customer simultaneously. For simplicity, the set of resource customers is represented by $C$, and $c_{i} \in C$ if $c_{i}$ acts as a customer. Likewise, the set of resource suppliers is illustrated by $S$, and $s_{j} \in S$ if $s_{j}$ acts as a supplier.

Once a resource customer $c_{i}$ needs resources or services, it takes turns asking its acquaintances $s_{j}\left(s_{j} \in S\right)$ for information. In this way, $c_{i}$ collects all resource supplier candidates. However, it is emphasized that $c_{i}$ has to assess the trustworthiness of these candidates in MARA with multiple potentially deceptive agents. In the following subsection, the proposed approach for trust estimation is described in detail.

\section{B. ITEM}

Typically, a customer $c_{i}\left(c_{i} \in C\right)$ estimates the trustworthiness of another resource supplier candidate $s_{j}\left(s_{j} \in S\right)$ with either direct trust or indirect reputation [6]. In this paper, both direct and indirect trust information are adopted.

It is necessary to emphasize that the trust information process involves much uncertainty caused by randomness, incompleteness, fading over time, and so on. Meanwhile, a customer might receive multiple recommendations provided by other clients, i.e., indirect reputation. Thus, the central issues for trustworthiness estimation lie in processing uncertainty and integrating multiple information [33]. We use DST to deal with these issues.

1) Generation of BPAs for direct trust: In this part, we discuss how to generate BPAs to express direct trust based on previous experience. Let $T$ mean that a customer agent considers a supplier agent to be trustworthy. Accordingly, $n T$ indicates that the supplier agent to be untrustworthy. Thus, the frame of discernment is $\Omega=\{T, n T\}$. the customer agent can generate evidence, i.e., BPAs, according to its previous experience to capture the reliability of the resource suppliers.

Firstly, we have to achieve the rates of satisfaction with the previous experience. Here, $S_{i j}(t)$ and $F_{i j}(t)$ represent respectively the rates of satisfaction and dissatisfaction of the supplier $s_{j}$ from the perspective of $c_{i}$ at time $t$. As stated, it is necessary to emphasize that the stability of memory fading over time, we have

$$
\begin{aligned}
& S_{i j}(t)=\sum_{r=1}^{R_{i j}}\left(e^{-\frac{R_{i j}-r+1}{\lambda_{i} * R_{i j}}} * \sin \left(\frac{[I(r)+1] \pi}{4}\right)\right) \\
& F_{i j}(t)=\sum_{r=1}^{R_{i j}}\left(e^{-\frac{R_{i j}-r+1}{\lambda_{i} * R_{i j}}} * \cos \left(\frac{[I(r)+1] \pi}{4}\right)\right)
\end{aligned}
$$

where $\lambda_{i}$ is the stability of memory to the customer $c_{i}$ (determines how fast a memory fading over time). $R_{i j}$ indicates the total number of interactions raised by the customer agent $c_{i}$ with the supplier agent $s_{j}$ and $r$ corresponds to the interaction sequence. $I(r)$ is the interactive outcome of the $r t h$ interaction, $I(r)=1$ if it is a positive feedback, otherwise, $I(r)=-1$.

Besides, in order to characterize uncertainty caused by incomplete information, randomness, etc., a fading factor $f_{i j}(t)$ is employed by the customer $c_{i}$. Then the BPA, namely the direct trust, which captures the reliability of the supplier $s_{j}$ by the customer $c_{i}$ at time $t$ is as follows,

$$
\left\{\begin{array}{l}
m_{i j}^{t \cdot d}(T)=S_{i j}(t) /\left(S_{i j}(t)+F_{i j}(t)\right) * f_{i j}(t) \\
m_{i j}^{t \cdot d}(n T)=F_{i j}(t) /\left(S_{i j}(t)+F_{i j}(t)\right) * f_{i j}(t) \\
m_{i j}^{t \cdot d}(T, n T)=1-\left(m_{i j}^{t \cdot d}(T)+m_{i j}^{t \cdot d}(n T)\right) .
\end{array}\right.
$$

The BPA can also be rewritten as $m_{i j}^{t \cdot d}=\left(m_{i j}^{t \cdot d}(T), m_{i j}^{t \cdot d}(n T)\right.$, $\left.m_{i j}^{t \cdot d}(T, n T)\right)$. As (3) and (4) exhibit, recent interactions have a greater impact on the estimation of direct trust. In this way, we modelled the fading property of direct experience. Moreover, this direct trust can be extended to dynamic MASs where agents have oscillating behaviors.

2) Modified Dempster's combination rule for indirect reputation: In the previous part, we studied direct trust according to a customer's previous experience. Generally, the customer would require indirect experience to make sound decisions. As stated in the preliminary part, the combination of conflicting evidence would result in inconsistent and irrational results. The inconsistent results would mislead the customer to choose a partner that is not trustworthy. In the literature [34], a method based on BPA preprocessing is proposed. Supposing that the customer $c_{i}$ has received $L$ BPAs $m_{q j}^{t \cdot i n d}(q=1, \ldots, L)$, each of which can be regarded as an indirect reputation provided by the third-party customer $c_{q}$. the pretreatment of these BPAs [34] is as following

$$
M A E(F)=\sum_{q=1}^{L} \omega_{q j} m_{q j}^{t \cdot i n d}(F)
$$

where $\omega_{q j}$ is the weighted degree of $m_{q j}^{t \cdot i n d}$ and $F$ is the element in the union of the focal elements in the $L$ BPAs. $M A E(F)$ represents the weighted average BPA of all the primitive evidence. After preprocessing, the classic Dempster's combination rule is used $L-1$ times to combine $M A E(F)$ to receive the final result [34]. However, it is challenging to decide the appropriate weight of $m_{q j}^{t \cdot i n d}$. In the previous methods [18], [23], [34], [35], especially outside the system of multiagents, evidence distance and entropy-based approaches and average weight approaches are dominant for data fusion. However, we recognize that the assigned weight is directly proportional to the entropy value [16], [17], [19], that is, the high weight is assigned to the evidence with high entropy, and 
it is not reasonable. Besides, the entropy of DST is rarely used alone for conflict evidence combination.

As explained, the generation of BPAs involves much uncertainty. Generally speaking, uncertainty can also be interpreted as the amount of information conveyed by the message, and it can be utilized to construct weights [24], [36]. Compared to the news "the sun rises from the east," it is accepted that "the sun rises from the west" carries more knowledge. Namely, when the data source produces a low-probability value, the event conducted more "information" than when a high-probability event occurs. Deng entropy shows the information contents contained by the BPAs (2). Therefore, the higher the entropy value, the greater the uncertainty the evidence is, and accordingly, the less information the evidence contains. Thus, lesser weight should be assigned. Moreover, the frame of decrement consists of two elements, i.e., $\Omega=\{T, n T\}$, considering the Deng entropy values as weights could be more accessible and acceptable. Suppose that $L$ BPAs $m_{q j}^{t \text {.ind }}(q=1, \ldots, L)$ are received by the customer $c_{i}$, then the detailed processes to fuse the $L$ indirect reputation are shown as follows,

(i) Compute Deng entropy of BPA $m_{q j}^{t \cdot \text { ind }}$ by (2) and results are represented by $E_{d}\left(m_{q j}^{t \cdot i n d}\right)$. It must be emphasized that because of the existence of uncertainty and fading functions, the evidence of $100 \%$ negative or positive does not exist, for instance, at time $t,\left(m_{q j}^{t \cdot \text { ind }}(T)=1\right.$, $\left.m_{q j}^{t \cdot i n d}(n T)=0, m_{q j}^{t \cdot i n d}(T, n T)=0\right)$ does not exist;

(ii) Normalize the Deng entropy $E_{d}\left(m_{q j}^{t \cdot i n d}\right)$, in order to observe obvious difference between the Deng entropy $E_{d}\left(m_{q j}^{t \cdot i n d}\right)$, we propose to use the equation below and results are represented by $\hat{E}_{d}\left(m_{q j}^{t \cdot i n d}\right)$

$$
\hat{E}_{d}\left(m_{q j}^{t \cdot i n d}\right)=\frac{\max _{k=1}^{L} e^{E_{d}\left(m_{k j}^{t \cdot i n d}\right)}-e^{E_{d}\left(m_{q j}^{t \cdot \text { ind }}\right)}}{\sum_{p=1}^{L}\left(\max _{k=1}^{L} e^{E_{d}\left(m_{k j}^{t \cdot \text {. } d ~}\right)}-e^{E_{d}\left(m_{p j}^{t \cdot \text {. nnd }}\right)}\right)}
$$

(iii) Afterward, each BPA is weighted with its normalized entropy, we therefore have $\omega_{q j}=\hat{E}_{d}\left(m_{q j}^{t \cdot i n d}\right)$ in (5). Thus, we modified the entropy-based weighted approach to combine multiple BPAs, that is, a great weight is appointed to the low-entropy BPA. From another perspective, agents tend to trust the third-party agents that provide more information in the trust estimation model, or third-party agents with higher certain indirect reputations are more trustworthy. If all the entropies are the same, the weights are set equally.

(iv) Finally, combine the modified BPA $L-1$ times as [34] stressed to obtain the final result.

In this way, the received $L$ BPAs are combined and the combined indirect reputation is represented by $\left(M_{i j}^{t \cdot i n d}(T)\right.$, $\left.M_{i j}^{t \cdot i n d}(n T), M_{i j}^{t \cdot i n d}(T, n T)\right)$.

3) Direct trust and indirect reputation combination: The customer $c_{i}$ therefore receives the direct trust and indirect reputation to the supplier $s_{j}$ at time $t$ with the beforementioned method, which are represented by $m_{i j}^{t \cdot d}$ and $M_{i j}^{t \cdot i n d}$ respectively. However, a customer usually relies more on its direct trust compared to the indirect reputation provided by

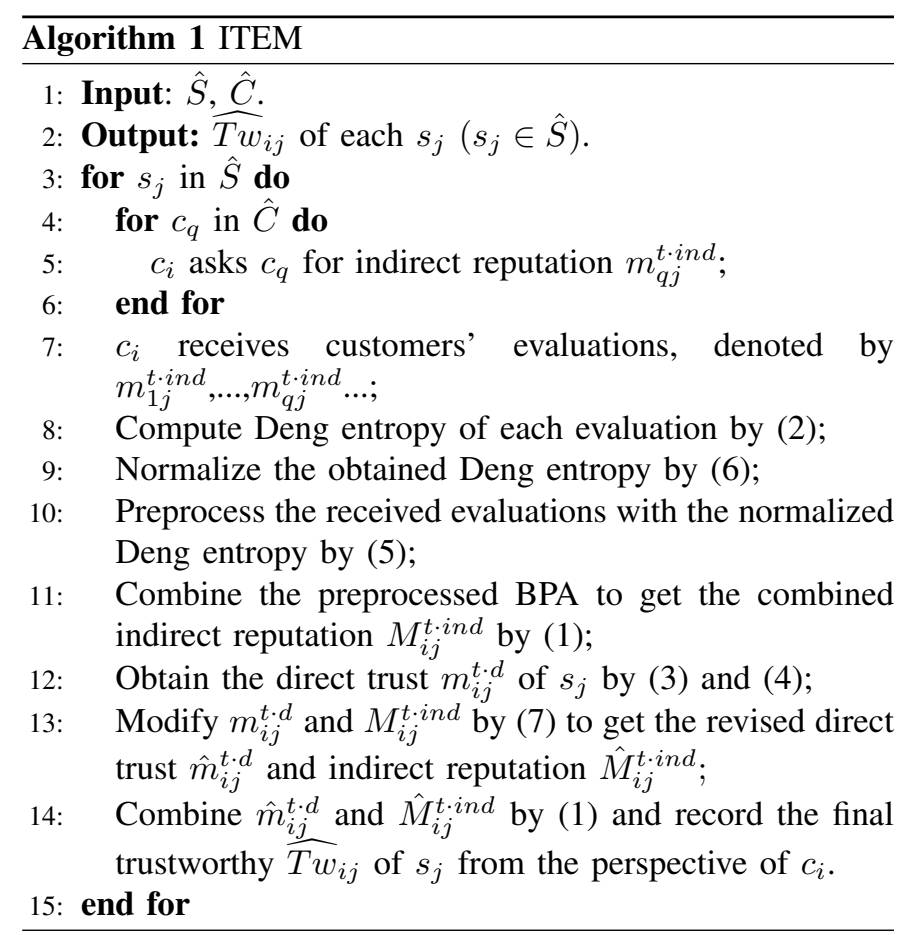

third-party agents. As a result, two weights, $W_{d}$ and $W_{\text {ind }}$, are employed to modify the direct trust $m_{i j}^{t \cdot d}$ and indirect reputation $M_{i j}^{t . i n d}$, respectively. The corresponding results after modification are represented by $\hat{m}_{i j}^{t \cdot d}$ and $\hat{M}_{i j}^{t \cdot i n d}$. The direct trust is revised as follows,

$$
\left\{\begin{array}{l}
\hat{m}_{i j}^{t \cdot d}(T)=m_{i j}^{t \cdot d}(T) * W_{d} \\
\hat{m}_{i j}^{t \cdot d}(n T)=m_{i j}^{t \cdot d}(n T) * W_{d} \\
\hat{m}_{i j}^{t \cdot d}(T, n T)=1-\left(\hat{m}_{i j}^{t \cdot d}(T)+\hat{m}_{i j}^{t \cdot d}(n T)\right) .
\end{array}\right.
$$

The indirect reputation is also revised in the same way by the weight of indirect reputation $W_{i n d}$. Afterward, the Dempster's combination rule is employed to fuse the modified direct trust and indirect reputation to get the ultimate trustworthiness $\widehat{T w}_{i j}$ of the supplier $s_{j}$ from the perspective of $c_{i}$. The general rule for the customer $c_{i}$ to estimate the trust of all the potential resource suppliers could be translated into the Algorithm 1, which is also named as Improved DST-basd Trust Estimation Model (ITEM). Our proposed ITEM has two inputs: $\hat{S}$ which is the set of prospective suppliers, and $\hat{C}$ is the set of thirdparty customers who provide indirect reputation to a certain supplier $s_{j}$. Specifically, for each potential resource supplier $s_{j} \in \hat{S}$, the customer $c_{i}$ receives recommendations from other customers (lines 4-6). Then indirect reputation is calculated with the proposed trust model (lines 7-11). Likewise, direct trust can be computed in the same way (line 12). Direct trust and indirect reputation are combined to obtain the ultimate trustworthy value of $s_{j}$ (lines 13-14). Finally, $c_{i}$ achieves the trust value of all suppliers.

\section{Resource reservation and resource allocation}

An agent in the proposed method makes decisions locally and prioritize its utility function. Therefore, a well-evaluated agent is more likely to be selected more than once. However, 
the allocation of resources may not be interrupted once they begin processing. Besides, the agent has no priority over other agents. Hence, we offer solutions guided by the concept of reservations to ensure a fair allocation of resources.

The customer $c_{i}\left(c_{i} \in C\right)$ attempts to reserve the required resource provided by the most reliable supplier $s_{j}\left(s_{j} \in S\right)$. $c_{i}$ sends a message to inform its unique identity and reservation time. The supplier $s_{j}$ acts generous and preserves the corresponding available resource. Once the exact resource is retained, no one has the right to assign it before the reservation period. In this way, the resource customer who first reserves the resource is prioritized to obtain the resource. "First come, first served" guarantees the fairness and normal operation of the proposed system.

\section{Simulation Study}

In this section, we conducted two separate simulations to illustrate the effectiveness of the proposed method. Firstly, the ITEM part of our proposed MARA-T\&R system in section IV-B is compared to beta reputation system (BRS) [4] in terms of the relative frequency of successful interactions (RFS) [37]. The comparison is employed to indicate the effectiveness of the ITEM for agents' trust estimation in MASs. Afterward, the proposed MARA-T\&R system is applied in a bicycle sharing system for resource allocation to test its effectiveness.

\section{A. ITEM vs. BRS}

Of the existing computational trust models, the BRS is widely applied in different fields and somehow similar to our trust method. It is a probabilistic model that relies on the number of successful and failed interactions [4]. We compare the performance in terms of the RFS, which evaluates the system based on how many interactions are executed to complete a target number of successful interactions. Specifically speaking, we exhibit the fraction of a fixed number of successful interactions over the total required amount of interactions. Here, it is necessary to state that a successful interaction for an agent corresponds to an interaction that selects it as a provider, and the latter has properly provided the service.

1) Simulation setting for ITEM vs. BRS: Our simulation uses one resource customer, 10 resource suppliers and 10 thirdparty resource customers, i.e., $\operatorname{card}(\hat{S})=\operatorname{card}(\hat{C})=10$. Each supplier $s_{j} \in \hat{S}$ has a constant trust value of providing satisfactory resources, which is sampled uniformly from the values of $0.1,0.2, \ldots, 0.9$. This value indicates the probability of a successful outcome when interacting with this supplier. For preprocessing, we let all the third-party customers interact with the resource suppliers so that they can achieve direct trust information about the suppliers. 5000 interactions are conducted to assure the data to be near-accurate, and each of the 5000 interactions a supplier $s_{j} \in \hat{S}$ is chosen randomly from the ten resource suppliers, and a third-party customer $c_{q}$ is randomly selected from the ten resource customers. Each resource customer $c_{q}$ remembers, for each resource supplier $s_{j}$, the interaction order, and the corresponding interactive feedback, which can be either success or failure.

Afterward, customers $c_{q} \in \hat{C}$ estimate their (direct) trust in any resource suppliers employing the previous information. Two different direct trust values, computed by the ITEM and the BRS are received respectively. In terms of the BRS, the customer $c_{q}$ models its trust in the supplier $s_{j}$ using the numbers of successful $p_{q j}$ and failed $n_{q j}$ interactions raised by $c_{q}$ with $s_{j}$. Then the trust value of $s_{j}$ from the perspective of $c_{q}$ can be represented by $\operatorname{br} s\left(p_{q j}, n_{q j}\right)=\frac{p_{q j}+1}{p_{q j}+n_{q j}+2}$ [4]. With regards to the ITEM, (3) and (4) are then adopted to generate the direct trust $m_{q j}^{t \cdot d}$. An honest third-party customer $c_{q}$ will simply report the actual evaluation when asked for direct experience with respect to the supplier $s_{j}$.

However, it must be emphasized that there are many deceptive agents in a MAS. In terms of the third-party resource customer settings, a partly customer firstly picks the resource suppliers for which it would provide distorted recommendations. Each supplier $s_{j}$ could be picked with a probability of $50 \%$. For all the other suppliers, $c_{q}$ would always be honest. If a supplier $s_{j}$ is in the list of providing distorted information, $c_{q}$ yields two random numbers $n_{q 1}$ and $n_{q 2}\left(n_{q 1}, n_{q 2} \in\right.$ $[0,1])$ with which it reports the distorted recommendation $m_{q j}^{t \cdot d}(T)=n_{q 1} * n_{q 2}, m_{q j}^{t \cdot d}(n T)=\left(1-n_{q 1}\right) * n_{q 2}$, and $m_{q j}^{t \cdot d}(T, n T)=1-m(T)-m(n T) . c_{q}$ also reports $n_{q 1} * H_{q}$ and $\left(1-n_{q 1}\right) * H_{q}$ rounded to 1 instead of the actual amounts of successful and failed interactions, where $H_{q}$ is the number of total interactions that $c_{q}$ has raised.

Subsequently, the resource customer $c_{i}$ tries to interact with a resource supplier chosen randomly from the 10 resource suppliers. $c_{i}$ starts with an empty set of direct trust, and then the experiment proceeds in rounds to reach a fixed number of successful interactions. Each round, we force $c_{i}$ to assess the trustworthiness only by indirect reputation provided by $c_{q}\left(c_{q} \in \hat{C}\right)$. When $c_{i}$ collected all indirect reputation at the selection step, two resource suppliers would be selected to conduct interactions based on the trustworthiness estimated by the ITEM and the BRS, respectively. Accordingly, we record the two selected suppliers and the corresponding feedback.

With regard to demonstrating the effectiveness of the revised entropy-based weighted approach for multiple BPAs combination, we use $\frac{e^{E_{d}\left(m_{q j}^{t \cdot i n d}\right)}}{\sum_{p=1}^{L} e^{E_{d}\left(m_{p j}^{t \cdot i n d}\right)}}$ to replace (6) in our proposed ITEM. As (6) shows, we hold that great weights should be appointed to the small-entropy BPAs. However, [16], [17], [19] employ $\frac{E_{d}\left(m_{q j}^{t \cdot i n d}\right)}{\sum_{p=1}^{L} E_{d}\left(m_{p j}^{t \cdot i n d}\right)}$, or $\frac{E_{d}\left(m_{q j}^{t \cdot i n d}\right)}{\max \left(E_{d}\left(m_{p j}^{t \cdot i n d}\right)\right.}$ for data fusion outside the system of multiagent. In other words, the weight assigned to BPA is proportional to the entropy value. The simulation terminates when the number of successful interactions reaches at 100 with the proposed method.

2) Results: Fig. 1 presents the RFSs obtained by the ITEM (blue cuboid) and the BRS (red cuboid) under $P \% P \in[0,50]$ dishonest agents, respectively. We ran 100 simulations and randomly select 10 times under 0 and $50 \%$ deceptive agents due to space constraints and similar results. As is shown in 


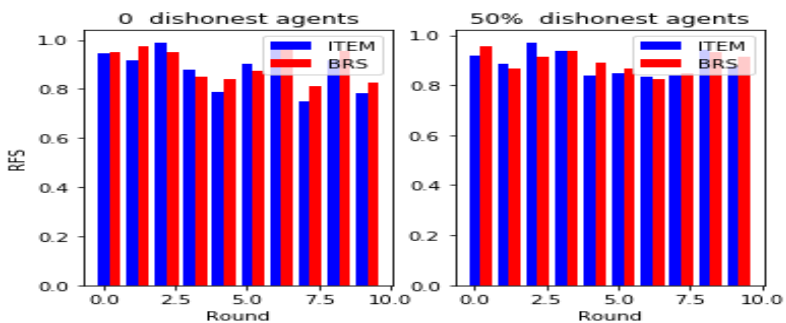

Fig. 1. the RFS comparison between the ITEM and the BRS

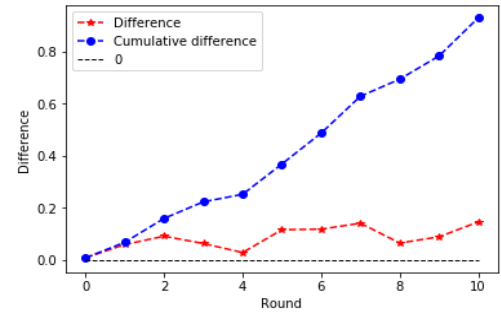

Fig. 2. RFS comparison of A) our proposed entropy-based weighted approach ITEM (high weight assigned to low-entropy BPAs) with B) the analogous version of ITEM with high weight assigned to high-entropy BPAs

Fig. 1, RFSs are nearly the same when several deception agents are involved in the MAS. That is to say, the ITEM part of our proposed MARA-T\&R system competes with the BRS for trust estimation. However, we have overcome the limitations in the context of the DST-based trust estimation models as discussed in the last paragraph of section II, from both generating BPAs and combing multiple BPAs aspects.

Fig. 2 exhibits the performances of the two entropy-based weighted approach for indirect reputation combination. We use the RFS value $v_{1}$ obtained by the proposed ITEM, minus the RFS value $v_{2}$ obtained by replacing (6) with $\frac{e^{E_{d}\left(m_{q j}^{t \cdot i n d}\right)}}{\sum_{p=1}^{L} e^{E_{d}\left(m_{p j}^{t \cdot i n d}\right)}}$ in ITEM. Therefore, each round, we receive a $v_{1}-v_{2}$, and the red line is hired to show the results. The blue line indicates the cumulative RFS differences. As displayed, we have $v_{1}-v_{2}>$ 0 in each round, which means the proposed entropy-based weighted approach could always receive a better RFS value. Thus, great weights assigned to low-entropy BPAs for multiple BPAs combination is correct.

In summary, the ITEM part of our proposed MARA-T\&R system is an ideal trust estimation model, and it is as efficient as BRS. However, there are at least three advantages in ITEM. First, we considered the fading property when generating evidence by previous experience ((3) and (4)), which has fulfilled the DST-based trust system. This approach is extendable for dynamic trust estimation. Secondly, we performed an information amount based approach to combine multiple indirect reputations, that have rarely been emphasized before in MASs for trust estimation. Lastly, we revised the entropybased weighted approaches for BPAs combination. That is, high weights should be assigned to small-entropy BPAs.

\section{B. Simulation setup of MARA T\&R system}

We also test the proposed MRAR-T\&R in an agent-based bike sharing system with the help of Python and modular Mesa. In the simulation setup, bicycles and user agents are placed in the grid ' $20 * 20$ ' randomly, where $(0,0)$ is assumed to be the bottom-left and $(19,19)$ is the top-right, more than one agent can be placed in the same cell.

In terms of resources (bicycles), they have identities, states, and locations, initialized by placing randomly in the grid. It is essential to emphasize that the status of the bicycle switches from 'Free,' 'Reserved,' and 'Busy.' A customer sends a reservation message to the available bicycle informing its identity and the reservation time. The state of the reserved bicycle converts to 'Busy' if the user arrives before the reservation time; otherwise, the reserved bicycle would become available ('Free') again. The "busy" bicycles become "Free" when reaches the destination.

In the simulation setup, a user agent has various properties and actions. These actions include "Update memory", 'Need bicycles,' 'Reserved a bicycle,' 'Using a bicycle' and 'Return a bicycle.' The user agent $a_{i}$ has two memories, namely mem $_{i}\left(\right.$ bike) and mem $_{i}$ (history), and both have limited lengths 20. mem $_{i}$ (bike) is used to record free bicycles seen in the same cell, which would act as resource information when others need a bicycle. mem $_{i}$ (history) remembers the interactive feedback, which acts as direct experiences for trust estimation. Each round, the user agent $a_{i}$ updates mem $_{i}($ bike) firstly. If $a_{i}$ needs a bike (with the probability of 0.3 ), it asks its neighbours (agents in the circle with a radius of 6 are regarded as neighbours) for information about available bicycles. We force the distance between its location and the destination to exceed $3 / 4$ times the length of the grid $([15,40]$ units of length), it is because we would like to compare the average waiting time. It is essential to stress that the distance of the cube $\left(x_{1}, y_{1}\right)$ and $\left(x_{2}, y_{2}\right)$ is calculated by $\left|x_{2}-x_{1}\right|+\left|y_{2}-y_{1}\right|$. In this circumstance, when the neighbour $a_{j}$ receives the message, it acts generously and provides its memory mem $_{j}($ bike). As stated, users in the system may provide misleading information, and it is reported that $31 \%$ person lies to an acquaintance in real life [38]. Accordingly, all user agents provide deceptive information with the probability satisfy the normal distribution with a mean equaling to 0.3 . Here, the misleading information is mainly to give a fake location of the shared bicycle by randomly selecting a neighbouring cell. When collected all the bike candidates, the user has to assess the trustworthiness of the information providers. Thus, the blackboard shares the collected information related to the supplier's pervious performance. The ITEM part of our proposed MARA-T\&R system in section IV-B is adopted for trust estimation, and $a_{i}$ selects and reserves a bicycle with the highest trust value and shortest distance. Afterward, the user agent goes towards the reserved shared bicycle. It is a success if it obtains the shared bicycle; otherwise, the interactive out-

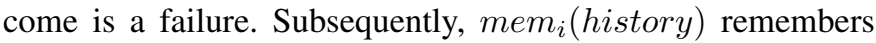
the interactive outcome and the corresponding information supplier. Simultaneously, the blackboard is real-time updated by collecting mem $_{i}$ (history). It would generously share the collected information as long as the other user agents need evidence for trust estimation. Finally, we calculate the average waiting time each round, which would be explained later. 


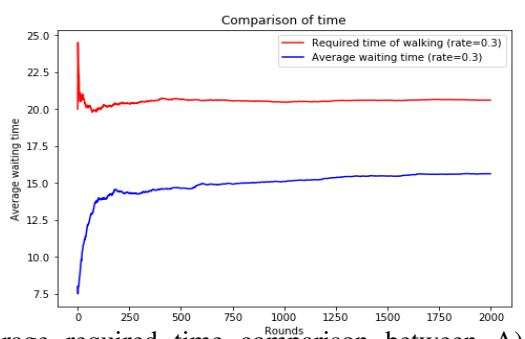

Fig. 3. Average required time comparison between A) trust is taken into consideration by the proposed ITEM (the red line), and B) Without considering trust (the blue line).

Each round, an experiment in the test environment is conducted using the parameters as explained. For example, the length and width of the cube are 20 units of length. There are 100 users and 100 bicycles in the network. Two things need to be stressed. Firstly, the waiting time starts from the time an agent needs a shared bicycle and ends when it reaches the destination. A user agent could either walk or ride to the destination. It moves forward three units of length per time with a bicycle. In contrast, an agent moves one unit of length if it walks. In this way, the distance can be interpreted as the required time walking from its position to the destination. However, if not characterized precisely, the misleading information provided by dishonest neighbouring agents would lead the user to a wrong place, which would waste more time than simply walking. We continue to calculate the waiting time of each agent until its request (to have a bike) is satisfied (in the worst case until its arrival at the destination). As a comparison, the distance is also recorded as it shows the time of walking to the destination. Secondly, the generation of basic probability assignment is conducted by calculating the satisfying degree of the historical interactions, and the fading factor is essential to generate BPAs. In this simulation setting, the generation of BPAs is defined in Equations 3 and 4 where $\lambda_{i}=0.25$ and $f_{i j}(t)=e^{-\frac{1}{R}}$. We run the simulation several times and Fig. 3 exhibits the results.

As is shown in Fig. 3, we could see that the average required time 15.8 noted by the red line (with trust) is much smaller than the average required time 20.70 represented by the blue line (without trust). That is to say, most user agents successfully find bicycles even if nearly $30 \%$ of neighbouring agents could be dishonest. More in detail, the average distance starts from nearly 7.5 in the first few rounds, as the blue line shows in Fig. 3. It is because there are plenty of free bicycles nearby, and a user has enough opportunity to receive a shared bicycle. However, the average time gradually increases as there are limited available resources (bicycles).

It has to be emphasized that 100 shared bicycles and 100 users are averagely placed in 400 cubes in the previous simulation. Meanwhile, some shared bicycles are reserved by other user agents which means that there are not enough available shared bicycles for users. Fig. 4 shows the comparison of the proportion of successfully obtaining a shared bicycle when there are enough bicycles (400 bicycles). Nevertheless, we also find that $61 \%$ of users received a shared bicycle successfully

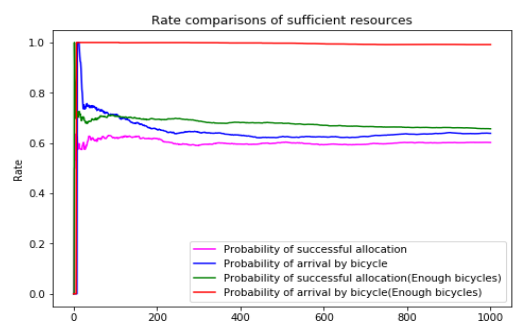

Fig. 4. Comparison of successful aundlocation each round between the green line: with sufficient resources and the magenta line: without sufficient resources; Comparison of reaching with a bicycle between the red line: with sufficient resources, and the blue line: with insufficient resources.

each round even without sufficient bicycles (as the magenta line shows in Fig. 4).

As exhibited in Fig. 4, the system provides enough shared bicycles (400 shared bicycle agents and 100 user agents). The average probability of providing misleading information satisfies the normal distribution with a mean equaling to 0.3 . Compared to the magenta line (with 100 bicycle agents), we find that the successful allocation rate indicated by the green line (with 400 bicycle agents) increased by nearly eight percent. Whereas, the improvement is not that much obvious. However, the ratio of riding to destinations could be around $98 \%$, which is a significant improvement. In other words, even without enough resources, the proposed MARA-T\&R system can find reliable partners. When with sufficient resources, the method can efficiently help obtain required resources.

\section{Discussion}

In the forenamed parts, we conducted two tests to illustrate the effectiveness of our proposed MARA-T\&R system. Firstly, we compared the ITEM part of our proposed MARA-T\&R system to BRS. Our ITEM competes with BRS with a small amount of dishonest agents involved. That is to say, the ITEM can be used for trust estimation in MASs. Moreover, at least three aspects have been improved from the perspectives of generating and combining evidence, which was detailed discussed at the end of section V-A. The improvements have overcome the shortcomings and also extended the DST-based trust model for dynamic trust estimation. Then we simulate our proposed MARA-T\&R system in a bike-sharing system. Because of the particular simulation setup, and no prior experience is provided, it is reasonable and convincing to compare the ratio of average waiting time and successful resource allocation to present the efficiency of the proposed method.

Generally speaking, the received evidence contains much uncertainty caused by many factors, including incomplete information, randomness and so forth. Thus, we use a fading factor to generate BPAs is reasonable. However, agents are used to acting stably. Therefore, the fading factor is only useful in generating a BPA, and it has little influence on the final results. In the next work, we would test the proposed method in a MAS where agents behave oscillating to show the power and significance of the fading factor. 


\section{CONCLUSION}

In this paper, we have provided two principal contributions. First, we have improved the DST-based trust model. In the previous literature of DST-based trust models in MASs, two issues were not well emphasized. One is about the reliability of evidence vanishing over time, and another is to cope with high-conflict evidence. We used the fading factor ((3) and (4)) as well as Deng entropy to improve these two shortcomings. The comparison results indicate that the proposed entropybased weight approach is correct and perfect in dealing with evidence combination. Meanwhile, the ITEM part of our proposed MARA-T\&R system can be extended for dynamic trust estimation in MASs. Second, we proposed a resource allocation system based on the trust of agents and the concept of reservation. We have then applied our model to a bicyclesharing system and the results showed its efficiency in bicycle selection in a MAS with deception agents.

However, we also have limitations that we will address in future work: First, we did not simulate the dynamic reliability of resource suppliers. The generated BPAs are sensitive to recent interactions, and the proposed trust model should work well with the dynamic change in the trustworthiness. Second, the proposed trust model does not work well in a system with a majority of dishonest agents. In the future, we would enhance our methods to overcome these limitations and solve more practical resource allocation problems.

\section{REFERENCES}

[1] Y. Chevaleyre, P. E. Dunne, U. Endriss, J. Lang, M. Lemaitre, N. Maudet, J. Padget, S. Phelps, J. A. Rodriguez-Aguilar, and P. Sousa, "Issues in multiagent resource allocation," Informatica, vol. 30, no. 1, 2006.

[2] S. P. Marsh, "Formalising trust as a computational concept," 1994.

[3] C.-J. Liau, "Belief, information acquisition, and trust in multi-agent systemsa modal logic formulation," Artificial Intelligence, vol. 149, no. 1, pp. 31-60, 2003.

[4] A. Jøsang and R. Ismail, "The beta reputation system," in Proceedings of the 15th bled electronic commerce conference, vol. 5, 2002, pp. 25022511.

[5] H. Yu, Z. Shen, C. Leung, C. Miao, and V. R. Lesser, "A survey of multi-agent trust management systems," IEEE Access, vol. 1, pp. 35$50,2013$.

[6] A. Jøsang, R. Ismail, and C. Boyd, "A survey of trust and reputation systems for online service provision," Decision support systems, vol. 43, no. 2, pp. 618-644, 2007.

[7] G. Xu, Z. Feng, H. Wu, and D. Zhao, "Swift trust in a virtual temporary system: A model based on the dempster-shafer theory of belief functions,' International Journal of Electronic Commerce, vol. 12, no. 1, pp. 93-126, 2007.

[8] R. Feng, X. Xu, X. Zhou, and J. Wan, "A trust evaluation algorithm for wireless sensor networks based on node behaviors and ds evidence theory," Sensors, vol. 11, no. 2, pp. 1345-1360, 2011.

[9] G. Pinhey, J. A. Doucette, and R. Cohen, "Using reservations for multiagent resource allocation with costly preemption," Multiagent and Grid Systems, vol. 14, no. 3, pp. 219-242, 2018.

[10] A. Beynier, N. Maudet, and A. Damamme, "Fairness in multiagent resource allocation with dynamic and partial observations," in Proceedings of the 17th International Conference on Autonomous Agents and MultiAgent Systems. International Foundation for Autonomous Agents and Multiagent Systems, 2018, pp. 1868-1870.

[11] A. P. Dempster, "Upper and lower probabilities induced by a multivalued mapping," Annals of Mathematical Statistics, vol. 38, no. 2, pp. 325339, 1967.

[12] G. Shafer, "A mathematical theory of evidence," A Mathematical Theory of Evidence, vol. 20, no. 1, p. 242, 1976.
[13] B. Yu and M. P. Singh, "Distributed reputation management for electronic commerce," Computational Intelligence, vol. 18, no. 4, pp. 535549,2002

[14] X. Qiu, L. Zhang, S. Wang, and G. Qian, "A trust transitivity model based-on dempster-shafer theory," Journal of Networks, vol. 5, no. 9, p. $1025,2010$.

[15] X. Deng, F. Xiao, and Y. Deng, "An improved distance-based total uncertainty measure in belief function theory," Applied Intelligence, vol. 46, no. 4, pp. 898-915, 2017.

[16] Y. Tang, D. Zhou, Z. He, and S. Xu, "An improved belief entropybased uncertainty management approach for sensor data fusion," International Journal of Distributed Sensor Networks, vol. 13, no. 7, p. $1550147717718497,2017$.

[17] Y. Tang, X. Fang, D. Zhou, and X. Lv, "Weighted deng entropy and its application in uncertainty measure," pp. 1-5, 2017.

[18] F. Liu, X. Gao, J. Zhao, and Y. Deng, "Generalized belief entropy and its application in identifying conflict evidence," IEEE Access, vol. 7, pp. 126625-126633, 2019.

[19] K. Yuan, F. Xiao, L. Fei, B. Kang, and Y. Deng, "Modeling sensor reliability in fault diagnosis based on evidence theory," Sensors, vol. 16, no. 1, p. $113,2016$.

[20] K. Wang, "A new multi-sensor target recognition framework based on dempster-shafer evidence theory." International Journal of Performability Engineering, vol. 14, no. 6, 2018.

[21] Y. Deng, "Generalized evidence theory," Applied Intelligence, vol. 43 no. 3, pp. 530-543, 2015.

[22] R. R. Yager, "On the dempster-shafer framework and new combination rules," Information sciences, vol. 41, no. 2, pp. 93-137, 1987.

[23] Y. Deng, W. Shi, Z. Zhu, and Q. Liu, "Combining belief functions based on distance of evidence," Decision support systems, vol. 38, no. 3, pp. 489-493, 2004.

[24] D. Han, Y. Deng, C. Han, and Z. Hou, "Weighted evidence combination based on distance of evidence and uncertainty measure," J. Infrared Millim. Waves, vol. 30, no. 5, pp. 396-400, 2011.

[25] Y. Deng, "Deng entropy: a generalized shannon entropy to measure uncertainty," viXra 1502.0222, 2015.

[26] L. Fei, Y. Deng, and S. Mahadevan, "Which is the best belief entropy," Journal of Latex Class Files, vol. 13, no. 9, pp. 1-4, 2015.

[27] D. Harmanec and G. J. Klir, "Measuring total uncertainty in dempstershafer theory: A novel approach," International journal of general system, vol. 22, no. 4, pp. 405-419, 1994.

[28] C. E. Shannon, "A mathematical theory of communication," ACM SIGMOBILE mobile computing and communications review, vol. 5, no. 1, pp. 3-55, 2001.

[29] P. Smets, "Information content of an evidence," International Journal of Man-Machine Studies, vol. 19, no. 1, pp. 33-43, 1983.

[30] R. R. Yager, "Entropy and specificity in a mathematical theory of evidence," International Journal of General System, vol. 9, no. 4, pp. 249-260, 1983.

[31] R. Jiroušek and P. P. Shenoy, "A new definition of entropy of belief functions in the dempster-shafer theory," International Journal of Approximate Reasoning, vol. 92, pp. 49-65, 2018.

[32] T. T. Doan and A. Olshevsky, "Distributed resource allocation on dynamic networks in quadratic time," Systems \& Control Letters, vol. 99, pp. 57-63, 2017.

[33] Y. Wang and M. P. Singh, "Formal trust model for multiagent systems." in IJCAI, vol. 7, 2007, pp. 1551-1556.

[34] Y. Deng, "A threat assessment model under uncertain environment," Mathematical Problems in Engineering, vol. 2015, 2015.

[35] J. Qian, X. Guo, and Y. Deng, "A novel method for combining conflicting evidences based on information entropy," Applied Intelligence, vol. 46, no. 4, pp. 876-888, 2017.

[36] G. J. Klir and H. W. Lewis, "Remarks on "measuring ambiguity in the evidence theory"," IEEE Transactions on Systems, Man, and Cybernetics-Part A: Systems and Humans, vol. 38, no. 4, pp. 995-999, 2008.

[37] H. Yu, Z. Shen, C. Miao, B. An, and C. Leung, "Filtering trust opinions through reinforcement learning," Decision Support Systems, vol. 66, pp. 102-113, 2014.

[38] U. Fischbacher and F. Föllmi-Heusi, "Lies in disguise-an experimental study on cheating," Journal of the European Economic Association, vol. 11 , no. 3 , pp. 525-547, 2013. 